\title{
Josephson effect in superconductive SNS heterostructures with barriers
}

\author{
Ozgur Cakir and Igor O. Kulik \\ Department of Physics, Bilkent University, Ankara 06533, Turkey \\ (Received 26 August 2002; revised manuscript received 19 March 2003; published 19 May 2003)
}

\begin{abstract}
The dc Josephson effect in a planar superconductor-normal-metal-superconductor (SNS) junction is studied in the existence of a $\delta$ barrier in the normal region. The Green function of the structure is obtained by solving the Gorkov equations for the structure and then the current is calculated from the Green functions. The effect of the strength and position of the barrier is investigated. The current shows a weak dependence on the position of the barrier and it is seen to be maximum when the barrier is at the middle of the normal region. Also it is found that the current shows a stronger dependence on the strength of the barrier at low temperatures. A comparative discussion of three possible types of Josephson junctions, the SIS, SCS, and SNS contacts, is presented.
\end{abstract}

DOI: 10.1103/PhysRevB.67.174514

PACS number(s): 74.50.+r, 74.45.+c, 74.25.Sv, 85.25.Cp

\section{INTRODUCTION}

Josephson effect is a well-known manifestation of macroscopic phase coherence, the mechanism of which is the phase coherent transport of electrons from one superconductor to another, in case when superconductors are weakly coupled to each other. ${ }^{1}$ Early studies of weak links were mainly on the SIS-type junctions (tunneling barriers). ${ }^{2,3}$ Another weakly coupled superconductive structure is the SCS contact (a direct metallic connection between superconductors through an orifice of diameter smaller than the coherence length). ${ }^{4} \mathrm{~A}$ new era in Josephson effect started with the discovery of a peculiar scattering mechanism at the SN boundary known as Andreev reflection. ${ }^{5}$ An electron in the normal region incident on the SN interface is reflected back as a hole, and vice versa, which may be interpreted as the condensation of the incident electron together with another electron, corresponding to the reflected hole, into a Cooper pair, or in the case of an incoming hole, as the disassociation of a Cooper pair. This initiated the study of the SNS-type weak links, first generations of which were by Kulik, ${ }^{6,7}$ Ishii, ${ }^{8}$ Bardeen and Johnson, ${ }^{9}$ Svidzinsky et al., ${ }^{10}$ and this new type of weak links brought a rich variety in physics and applications of Josephson effect. ${ }^{11-13}$ In SNS structures, the dc Josephson effect arises due to coherent charge transport in the normal region, the mechanism of which is Andreev reflection, which may be seen as the transport of Cooper pairs from one superconducting electrode to another. ${ }^{11-13}$

Most of the authors calculated current using Green functions and the main concern was the construction of Green functions, which came out to be intriguing and complicated. ${ }^{6,8}$ McMillan's method of Green-function construction, though he intended to explain Tomasch oscillations in $\mathrm{SN}$ contact, proved to be very useful in SNS junctions. ${ }^{14}$ In this approach, the Green functions are obtained from the scattering solutions, i.e., from the solutions of the Bogoliubov-de Gennes equations. ${ }^{15}$ By extending this construction, more recently, Furusaki and Tsukada obtained a Landauer-type formula for the dc Josephson current. ${ }^{11,12}$

In real contacts, $S$ and $N$ metals are not identical, and impurities and imperfections in the metal bulk as well as on their interface make non-Andreev scattering possible. In this paper we investigate how these two scattering mechanisms (the normal reflection and the Andreev or retroreflection) cooperate. The non-Andreev reflection reduces the magnitude of the supercurrent and changes its temperature and phase dependence.

In our paper, a planar SNS structure with a stepwise pair potential and a $\delta$ barrier within the normal region is considered as a model compromising between the Andreev and the non-Andreev scatterings. The Green function for the junction with a barrier inside the normal metal is calculated by solving the Gorkov equations in a quasiclassical approximation ${ }^{16}$ with the appropriate boundary conditions, and from the Green functions the Josephson current is found. As the barrier strength is taken to zero, the pure SNS case, and as the width of the normal region is taken to be zero, the SIS case is recovered.

Similar, but not equivalent, problems of potential scattering in Josephson junctions of the SN (in fact SIN) type with smooth potentials have been considered by Zaitsev ${ }^{17}$ in the clean limit and by Golubov et al., ${ }^{19}$ Kuprianov and Lukichev $^{18}$ in the dirty limit. They derived appropriate boundary conditions for the Green functions to the left $(x$ $\ll \delta)$ and to the right $(x \gg \delta)$ of the barrier, where $\delta$ is the de Broglie wavelength of the Fermi electron, $\delta \sim \hbar / p_{F}$. Our case will be when the barrier is deep inside the normal metal in the SNS contact (i.e., a SNINS contact); in that case, the assumption of sharp potential is just the way of introducing consistently the effect of electron reflection inside the normal metal, which results in a non-Andreev reflection and in decrease in Josephson current through the junction.

Gorkov formulation of superconductivity introduces two Green functions in the Matsubara finite-temperature technique, $G_{\omega}\left(\mathbf{r}, \mathbf{r}^{\prime}\right)$ and $F_{\omega}^{\dagger}\left(\mathbf{r}, \mathbf{r}^{\prime}\right)$, and the pair potential $\Delta(\mathbf{r})$ satisfying the self-consistent equation, which in case of pair potential varying only in one dimension read ${ }^{20}$

$$
\begin{aligned}
& \quad\left[i \omega-T_{x}-U(x)\right] G_{\omega}\left(x, x^{\prime} ; k_{\perp}\right)+\Delta(x) F_{\omega}^{+}\left(x, x^{\prime} ; k_{\perp}\right) \\
& \quad=\delta\left(x-x^{\prime}\right) \\
& \left(i \omega+T_{x}+U(x)\right) F_{\omega}^{+}\left(x, x^{\prime} ; k_{\perp}\right)+\Delta^{*}(x) G_{\omega}\left(x, x^{\prime} ; k_{\perp}\right)=0,
\end{aligned}
$$




$$
\Delta^{*}(x)=|\lambda| \sum_{\omega} F_{\omega}^{+}(x, x)
$$

where $\omega=(2 n+1) \pi T$, with $n=0, \pm 1, \pm 2, \ldots \quad$ and $G_{\omega}\left(x, x^{\prime} ; k_{\perp}\right)$ and $F_{\omega}^{+}\left(x, x^{\prime} ; k_{\perp}\right)$ are the Fourier transforms,

$$
\begin{aligned}
& G_{\omega}\left(\mathbf{r}, \mathbf{r}^{\prime}\right)=\frac{1}{(2 \pi)^{2}} \int d^{2} k_{\perp} G_{\omega}\left(x, x^{\prime} ; k_{\perp}\right) e^{i \mathbf{k}_{\perp}\left(\mathbf{r}-\mathbf{r}^{\prime}\right)} \\
& F_{\omega}^{+}\left(\mathbf{r}, r^{\prime}\right)=\frac{1}{(2 \pi)^{2}} \int d^{2} k_{\perp} F_{\omega}^{+}\left(x, x^{\prime} ; k_{\perp}\right) e^{i \mathbf{k}_{\perp}\left(\mathbf{r}-\mathbf{r}^{\prime}\right)},
\end{aligned}
$$

and $\mathbf{k}_{\perp}$ is the transverse momentum. The Josephson current can be calculated from the single-particle Green function $G_{\omega}\left(\mathbf{r}, \mathbf{r}^{\prime}\right)$ as

$$
\begin{aligned}
\mathbf{J} & =\left.\frac{i e T}{m} \sum_{\omega}\left(\nabla^{\prime}-\nabla\right) G_{\omega}\left(\mathbf{r}, \mathbf{r}^{\prime}\right)\right|_{\mathbf{r}^{\prime} \rightarrow \mathbf{r}} \\
& =\left.\hat{x} \frac{i e T}{m(2 \pi)^{2}} \sum_{\omega} \int d^{2} k_{\perp}\left(\partial_{x^{\prime}}-\partial_{x}\right) G_{\omega}\left(x, x^{\prime} ; k_{\perp}\right)\right|_{x^{\prime} \rightarrow x} .
\end{aligned}
$$

Once we know the single-particle Green function we can calculate the Josephson current.

\section{QUASICLASSICAL TREATMENT OF GORKOV EQUATIONS}

In our approach we will make use of quasiclassical Green-function formulation of superconductivity, i.e., the condition that characteristic de Broglie wavelength of an electron $\lambda_{F}=2 \pi / k_{F} \sim 10^{-8} \mathrm{~cm}$ is much smaller than the characteristic length at which the pair potential changes, where the coherence length of a superconductor $\xi \sim \hbar v_{F} / \Delta$ which is of the order of $10^{-5}-10^{-6} \mathrm{~cm}$ for $s$-wave superconductors. Since there is normal scattering of quasiparticles due to the barrier, the Fermi momentum is not conserved throughout the structure. Hence quasiclassical approximation can only be implemented within the regions where there is no normal scattering, with the scattering at short-length barriers considered via appropriate boundary conditions for quasiclassical Green functions.

The very essence of the semiclassical treatment is the elimination of fast varying terms at the Fermi wavelength in comparison with superconductive quantities that vary at distances of the order of the coherence length. ${ }^{16}$ In the context of semiclassical treatment, the Green functions are decomposed into two parts,

$$
\mathcal{G}_{\omega}\left(x, x^{\prime} ; k_{\perp}\right)=\sum_{\sigma= \pm} g_{\omega}^{\sigma}\left(x, x^{\prime} ; k_{\perp}\right) e^{i \sigma p x},
$$

where

$$
\mathcal{G}_{\omega}=\left(\begin{array}{c}
G_{\omega} \\
F_{\omega}^{+}
\end{array}\right), \quad g_{\omega}=\left(\begin{array}{l}
g_{\omega} \\
f_{\omega}
\end{array}\right)
$$

and $p=\sqrt{2 m \mu-k_{\perp}^{2}}$ is the $x$ component of the Fermi momentum. Except those points at which the derivative of the Green function may suffer discontinuities, semiclassical forms (5) inserted into the Gorkov equations, upon keeping terms of the first order in $\lambda_{F} / \xi$ and ignoring higher order terms, yield the equations for the semiclassical Green functions (with $\partial_{x}$ $=\partial / \partial x$ and $v=p / m$ )

$$
\left(\begin{array}{cc}
i \omega+i \sigma v \partial_{x} & \Delta(x) \\
\Delta *(x) & i \omega-i \sigma v \partial_{x}
\end{array}\right) g_{\omega}=0 .
$$

equipped with the necessary boundary conditions. These conditions will be derived in the following section. Equation (6) is satisfied in a region where the derivative of the Green function does not suffer any discontinuities. The semiclassical Green functions $g_{\omega}$ no longer have fast oscillations as a function of $x$. Elimination of fast varying terms reduces the order of the differential Gorkov equations from 2 to 1 .

In a superconducting region with a pair potential $\Delta(x)$ $=\Delta e^{i \varphi}$ where there is no normal scattering, the solutions of the semiclassical equations (6) consist of the superposition of the two terms,

$$
\begin{aligned}
g_{\omega}^{\sigma}= & \alpha\left(x^{\prime}\right)\left(\begin{array}{c}
e^{i \varphi / 2} \\
-i \frac{\omega+\sigma \Omega}{\Delta} e^{-i \varphi / 2}
\end{array}\right) e^{\left(\sqrt{\omega^{2}+\Delta^{2}} / v\right) x}+\beta\left(x^{\prime}\right) \\
& \times\left(\begin{array}{c}
e^{i \varphi / 2} \\
-i \frac{\omega-\sigma \Omega}{\Delta} e^{-i \varphi / 2}
\end{array}\right) e^{-\left(\sqrt{\left.\omega^{2}+\Delta^{2} / v\right) x}\right.}
\end{aligned}
$$

whereas in the normal region

$$
g_{\omega}^{\sigma}=\alpha\left(x^{\prime}\right)\left(\begin{array}{l}
1 \\
0
\end{array}\right) e^{-\sigma \omega x / v}+\beta\left(x^{\prime}\right)\left(\begin{array}{l}
0 \\
1
\end{array}\right) e^{\sigma \omega x / v},
$$

where $v=\sqrt{2 m \mu-k_{\perp}^{2}} / m$ is the Fermi velocity in the $x$ direction. The solutions in the different regions $(x<-d / 2$, $-d / 2<x<x^{\prime}, x^{\prime}<x<d / 2, d / 2<x$, where $d$ is the thickness of the normal metal) is written down in the form of linear combinations, as shown above, and then boundary conditions to be specified are imposed, allowing us to find the coefficients, thus obtaining the full Green functions. This recipe will be specified in the following section in which the Green function is obtained for an SNS structure with a $\delta$ barrier.

\section{SNS STRUCTURE WITH A $\delta$ BARRIER}

In this section we are going to consider an SNS structure with a $\delta$ barrier within the normal region, and a stepwise pair potential $\Delta(x)$ (see Fig. 1), i.e.,

$$
\Delta(x)= \begin{cases}\Delta e^{-i \varphi / 2} & \text { at } x<-d / 2 \\ 0 & \text { at }-d / 2<x<d / 2 \\ \Delta e^{i \varphi / 2} & \text { at } x>d / 2\end{cases}
$$

where the barrier potential

$$
U(x)=V \delta(x-a) \text { with }-d / 2<a<d / 2 \text {. }
$$




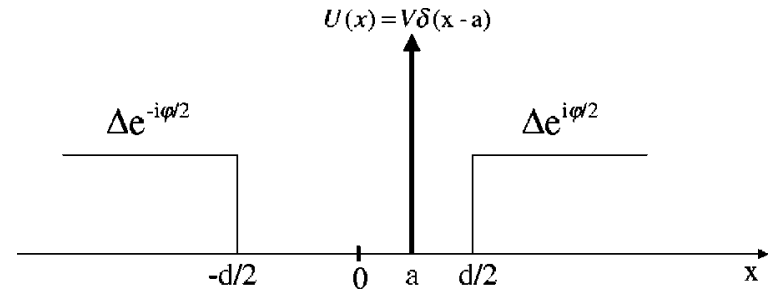

FIG. 1. SNINS structure modeled by a stepwise pair potential of phase difference $\varphi$ with a delta barrier $U(x)=V \delta(x-a)$ in the normal region.

The derivative of the Green function has jumps at points $x$ $=a$ and at $x=x^{\prime}$, whereas at any other $x$ the Green function and its derivative are continuous. The boundary conditions at the $\mathrm{SN}$ interface are

$$
\begin{gathered}
\mathcal{G}_{\omega}\left(x+0, x^{\prime}\right)=\left.\mathcal{G}_{\omega}\left(x-0, x^{\prime}\right)\right|_{x= \pm d / 2}, \\
\left.\partial_{x} \mathcal{G}_{\omega}\left(x, x^{\prime}\right)\right|_{x= \pm d / 2+0}=\left.\partial_{x} \mathcal{G}_{\omega}\left(x, x^{\prime}\right)\right|_{x= \pm d / 2-0} .
\end{gathered}
$$

Within the semiclassical approximation, these two conditions are equivalent and reduce to

$$
g_{\omega}^{\sigma}\left(x+0, x^{\prime}\right)=\left.g_{\omega}^{\sigma}\left(x-0, x^{\prime}\right)\right|_{x= \pm d / 2} .
$$

At $x=x^{\prime}$, the Green functions are continuous,

$$
\left.\mathcal{G}_{\omega}\left(x, x^{\prime}\right)\right|_{x=x^{\prime}-0} ^{x=x^{\prime}+0}=\left.0 \rightarrow \sum_{\sigma} e^{i \sigma p x} g_{\omega}^{\sigma}\left(x, x^{\prime}\right)\right|_{x=x^{\prime}-0} ^{x=x^{\prime}+0}=0
$$

where the vertical bar introduces the difference between the values at $x=x^{\prime}+0$ and $x=x^{\prime}-0$, whereas the derivative of the single-particle Green function has a jump,

$$
\begin{aligned}
\left.\partial_{x} G_{\omega}\left(x, x^{\prime}\right)\right|_{x=x^{\prime}-0} ^{x=x^{\prime}+0}= & 2 m \rightarrow \sum_{\sigma} e^{i \sigma p x}\left(i \sigma p+\partial_{x}\right) \\
& \times\left. g_{\omega}^{\sigma}\left(x, x^{\prime}\right)\right|_{x=x^{\prime}-0} ^{x=x^{\prime}+0} \\
= & \left.2 m \rightarrow \sum_{\sigma} e^{i \sigma p x} \sigma g_{\omega}^{\sigma}\left(x, x^{\prime}\right)\right|_{x=x^{\prime}-0} ^{x=x^{\prime}+0} \\
& =-2 i / v .
\end{aligned}
$$

Here the semiclassical forms are substituted according to Eq. (5), and the semiclassical approximation is applied such that the $\partial_{x} g_{\omega}$ term is discarded in comparison with the term containing $i \sigma p$. At the same time the derivative of the Gorkov Green function remains continuous at $x=x^{\prime}$,

$$
\left.\partial_{x} F_{\omega}^{+}\left(x, x^{\prime}\right)\right|_{x=x^{\prime}-0} ^{x=x^{\prime}+0}=\left.0 \rightarrow \sum_{\sigma} e^{i \sigma p x} \sigma f_{\omega}^{\sigma}\left(x, x^{\prime}\right)\right|_{x=x^{\prime}-0} ^{x=x^{\prime}+0}=0
$$

and again the semiclassical forms are substituted. The Green functions are continuous at the barrier location, $x=a$,

$$
\left.\mathcal{G}\left(x, x^{\prime}\right)\right|_{x=a-0} ^{x=a+0}=\left.0 \rightarrow \sum_{\sigma} e^{i \sigma p x} g_{\omega}^{\sigma}\left(x, x^{\prime}\right)\right|_{x=a-0} ^{x=a+0}=0
$$

whereas the derivative of the Green functions has a jump due to the $\delta$ barrier,

$$
\begin{aligned}
\left.\partial_{x} \mathcal{G}_{\omega}\left(x, x^{\prime}\right)\right|_{x=a-0} ^{x=a+0} & =2 m V \mathcal{G}_{\omega}\left(a, x^{\prime}\right) \\
& \left.\rightarrow \sum_{\sigma} e^{i \sigma p x}\left(i \sigma p+\partial_{x}\right) g_{\omega}^{\sigma}\left(x, x^{\prime}\right)\right|_{x=a-0} ^{x=a+0} \\
= & 2 m V \mathcal{G}_{\omega}\left(a, x^{\prime}\right) \\
\rightarrow & \sum_{\sigma}\left\{\left.e^{i \sigma p x} \sigma g_{\omega}^{\sigma}\left(x, x^{\prime}\right)\right|_{x=a-0} ^{x=a+0}\right. \\
& \left.+2 i Z e^{i \sigma p a} g_{\omega}^{\sigma}\left(a, x^{\prime}\right)\right\}=0
\end{aligned}
$$

where $Z=V / v$ is the dimensionless barrier strength.

Now we are going to write down the solutions in the five regions and then implement the boundary conditions. Given $-d<x^{\prime}<d$, the solutions are written as follows

$$
\begin{aligned}
& \text { At } x<-d / 2, \quad g_{\omega}^{\sigma}=A_{\sigma}\left(x^{\prime}\right)\left(\begin{array}{c}
e^{-i \varphi / 4} \\
-i \frac{\omega+\sigma \Omega}{\Delta} e^{i \varphi / 4}
\end{array}\right) e^{(\Omega / v) x} \\
& \text { At }-d / 2<x<x^{\prime}, \quad g_{\omega}^{\sigma}=\left(\begin{array}{c}
B_{\sigma}\left(x^{\prime}\right) e^{-\sigma(\omega / \nu) x} \\
C_{\sigma}\left(x^{\prime}\right) e^{\sigma(\omega / \nu) x}
\end{array}\right) \\
& \text { At } x^{\prime}<x<a, \quad g_{\omega}^{\sigma}=\left(\begin{array}{c}
D_{\sigma}\left(x^{\prime}\right) e^{-\sigma(\omega / \nu) x} \\
F_{\sigma}\left(x^{\prime}\right) e^{\sigma(\omega / \nu) x}
\end{array}\right) \\
& \text { At } a<x<d / 2, \quad g_{\omega}^{\sigma}=\left(\begin{array}{c}
G_{\sigma}\left(x^{\prime}\right) e^{-\sigma(\omega / \nu) x} \\
H_{\sigma}\left(x^{\prime}\right) e^{\sigma(\omega / \nu) x}
\end{array}\right)
\end{aligned}
$$

At $d / 2<x, \quad g_{\omega}^{\sigma}=J_{\sigma}\left(x^{\prime}\right)$

$$
\times\left(\begin{array}{c}
e^{i \varphi / 4} \\
-i[(\omega-\sigma \Omega) / \Delta] e^{-i \varphi / 4}
\end{array}\right) e^{-(\Omega / v) x}
$$

where $\Omega=\sqrt{\omega^{2}+\Delta^{2}}$. All 16 coefficients $A_{\sigma}, B_{\sigma}, C_{\sigma}, D_{\sigma}$, $F_{\sigma}, G_{\sigma}, H_{\sigma}, J_{\sigma}(\sigma= \pm)$ are functions of $x^{\prime}$.

Now the boundary conditions have to be imposed. At $x$ $=-d / 2$, boundary conditions (12) yield

$$
\begin{gathered}
B_{+} e^{(\omega / \nu) d / 2}-A_{+} e^{-(\Omega / \nu) d / 2-i \varphi / 4}=0, \\
C_{+} e^{-(\omega / \nu) d / 2}-A_{+} \gamma^{+} e^{-(\Omega / \nu) d / 2+i \varphi / 4}=0, \\
B_{-} e^{-(\omega / \nu) d / 2}-A_{-} e^{-(\Omega / \nu) d / 2-i \varphi / 4}=0, \\
C_{-} e^{(\omega / \nu) d / 2}-A_{-} \gamma^{-} e^{-(\Omega / \nu) d / 2+i \varphi / 4}=0,
\end{gathered}
$$

where $\gamma^{ \pm}=-i(\omega \pm \Omega) / \Delta$. At $x=x^{\prime}$, boundary conditions (13)-(15) are

$$
\begin{gathered}
\left(D_{+}-B_{+}\right) e^{(i p-\omega / \nu) x^{\prime}}+\left(D_{-}-B_{-}\right) e^{(-i p+\omega / \nu) x^{\prime}}=0 \\
\left(D_{+}-B_{+}\right) e^{(i p-\omega / \nu) x^{\prime}}-\left(D_{-}-B_{-}\right) e^{(-i p+\omega / \nu) x^{\prime}}=-2 i / v
\end{gathered}
$$




$$
\begin{aligned}
& \left(F_{+}-C_{+}\right) e^{(i p+\omega / \nu) x^{\prime}}+\left(F_{-}-C_{-}\right) e^{(-i p-\omega / \nu) x^{\prime}}=0 \\
& \left(F_{+}-C_{+}\right) e^{(i p+\omega / \nu) x^{\prime}}-\left(F_{-}-C_{-}\right) e^{(-i p-\omega / \nu) x^{\prime}}=0 .
\end{aligned}
$$

At $x=a$, boundary conditions (16) and (17) apply,

$$
\begin{gathered}
\left(G_{+}-D_{+}\right) e^{(i p-\omega / \nu) a}+\left(G_{-}-D_{-}\right) e^{(-i p+\omega / \nu) a}=0, \\
{\left[G_{+}-(1-2 i Z) D_{+}\right] e^{(i p-\omega / \nu) a}} \\
-\left[G_{-}-(1+2 i Z) D_{-}\right] e^{(-i p+\omega / \nu) a}=0, \\
\left(H_{+}-F_{+}\right) e^{(i p+\omega / \nu) a}+\left(H_{-}-F_{-}\right) e^{(-i p-\omega / \nu) a}=0, \\
{\left[H_{+}-(1-2 i Z) F_{+}\right] e^{(i p+\omega / \nu) a}} \\
-\left[H_{-}-(1+2 i Z) F_{-}\right] e^{(-i p-\omega / \nu) a}=0,
\end{gathered}
$$

and finally, at $x=d / 2$ boundary conditions (12) and (13) result in the identities

$$
J_{+} e^{-(\Omega / \nu) d / 2+i \varphi / 4}-G_{+} e^{-(\omega / \nu) d / 2}=0,
$$

$$
\begin{gathered}
J_{+} \gamma^{-} e^{-(\Omega / \nu) d / 2-i \varphi / 4}-H_{+} e^{(\omega / \nu) d / 2}=0, \\
J_{-} e^{-(\Omega / \nu) d / 2+i \varphi / 4}-G_{-} e^{(\omega / \nu) d / 2}=0,
\end{gathered}
$$$$
J_{-} \gamma^{+} e^{-(\Omega / \nu) d / 2-i \varphi / 4}-H_{-} e^{-(\omega / \nu) d / 2}=0,
$$

which make in totality the 16 equations needed for 16 unknowns.

We do not need to know all the coefficients when we wish to find the Josephson current. According to the current expression, Eq. (3), we only need the single-particle Green function for $x$ and $x^{\prime}$ in the same region, which will be chosen to be $a>x>x^{\prime}>-d$. In this region, only the $D_{\sigma}$ coefficients are needed for the Green function,

$$
G_{\omega}\left(x, x^{\prime} ; k_{\perp}\right)=\sum_{\sigma} D_{\sigma} e^{i \sigma(p-\omega / v) x} .
$$

Solving the equations for the boundaries thus obtaining the coefficients $D_{+}, D_{-}$, the Green function is written as

$$
\begin{aligned}
G_{\omega}= & \frac{i}{v}\left\{\left(1+Z^{2}\right)\left(\gamma^{+} e^{(\omega / \nu) d-i \varphi / 2}-\gamma^{-} e^{-(\omega / \nu) d+i \varphi / 2}\right)\left(\gamma^{+} e^{(\omega / \nu) d+i \varphi / 2}-\gamma^{-} e^{-(\omega / \nu) d-i \varphi / 2}\right)+Z^{2}\left(e^{i \varphi / 2+2(\omega / \nu) a}\right.\right. \\
& \left.\left.-e^{-i \varphi-2(\omega / \nu) a}\right)\left(e^{i \varphi / 2-2(\omega / \nu) a}-e^{-i \varphi / 2+2(\omega / \nu) a}\right)\right\}^{-1}\left\{\left[-\left(1+Z^{2}\right) \gamma^{+} e^{i \varphi / 2+(\omega / \nu) d}\left(\gamma^{+} e^{(\omega / \nu) d-i \varphi / 2}-\gamma^{-} e^{-(\omega / \nu) d+i \varphi / 2}\right)\right.\right. \\
& \left.-Z^{2} e^{i \varphi / 2+2(\omega / \nu) a}\left(e^{i \varphi / 2-2(\omega / \nu) a}-e^{-i \varphi / 2+2(\omega / \nu) a}\right)\right] e^{(i p-(\omega / \nu))\left(x-x^{\prime}\right)}+\left[-\gamma^{-}\left(1+Z^{2}\right) e^{i \varphi / 2-(\omega / \nu) d}\left(\gamma^{+} e^{i \varphi / 2+(\omega / \nu) d}\right.\right. \\
& \left.\left.\left.-\gamma^{-} e^{-i \varphi / 2-(\omega / \nu) d}\right)+Z^{2} e^{i \varphi / 2-2(\omega / \nu) a}\left(e^{i \varphi / 2+2(\omega / \nu) a}-e^{-i \varphi / 2-2(\omega / \nu) a}\right)\right] e^{-(i p-(\omega / \nu))\left(x-x^{\prime}\right)}\right\}+f\left(x+x^{\prime}\right) .
\end{aligned}
$$

There is an extra term $f\left(x+x^{\prime}\right)$ that does not contribute to the current since it is a function of $x+x^{\prime}$ that vanishes upon substitution into Eq. (3). The Green function can be decomposed into two parts:

$$
G_{\omega}\left(x, x^{\prime} ; k_{\perp}\right)=G_{\omega}^{0}\left(x, x^{\prime} ; k_{\perp}\right)+G_{\omega}^{1}\left(x, x^{\prime} ; k_{\perp}\right)
$$

where $G_{\omega}^{0}\left(x, x^{\prime} ; k_{\perp}\right)$ is the Green function of a normal metal with a $\delta$ barrier $V \delta(x-a)$ located at $x=a$, and $G_{\omega}^{1}$ is the contribution arising due to onset of steplike gap potential (9). In the interval $a>x>x^{\prime}$, the normal-metal Green function is

$$
G_{\omega}^{0}\left(x, x^{\prime} ; k_{\perp}\right)=-\frac{i}{v} \operatorname{sgn} \omega e^{(i p \operatorname{sgn} \omega-|\omega| / v)\left(x-x^{\prime}\right)}-\frac{1}{v} \frac{Z}{1+i Z \operatorname{sgn} \omega} e^{(-i p \operatorname{sgn} \omega+|\omega| / v)\left(x+x^{\prime}-2 a\right)} .
$$

Component $G_{\omega}^{1}\left(x, x^{\prime} ; k_{\perp}\right)$ at $a>x>x^{\prime}>-d / 2$, making a first-order contribution to the current in powers of $\lambda_{F} / \xi$, is

$$
\begin{aligned}
G_{\omega}^{1}\left(x, x^{\prime} ; k_{\perp}\right)= & \frac{i}{v}\left[\left(1+Z^{2}\right)\left(\gamma^{+} e^{i \varphi / 2+(\omega / \nu) d}-\gamma^{-} e^{-i \varphi / 2-(\omega / \nu) d}\right)\left(\gamma^{+} e^{-i \varphi / 2+(\omega / \nu) d}-\gamma^{-} e^{i \varphi / 2-(\omega / \nu) d}\right)+Z^{2}\left(e^{i \varphi / 2+2(\omega / \nu) a}\right.\right. \\
& \left.\left.-e^{-i \varphi / 2-2(\omega / \nu) a}\right)\left(e^{i \varphi / 2-2(\omega / \nu) a}-e^{-i \varphi / 2+2(\omega / \nu) a}\right)\right]^{-1}\left\{\left[-\gamma^{-\operatorname{sgn} \omega}\left(1+Z^{2}\right)\left(\gamma^{+} e^{-i \varphi / 2+(\omega / \nu) d}\right.\right.\right. \\
& \left.\left.-\gamma^{-} e^{i \varphi / 2-(\omega / \nu) d}\right) e^{-(i \varphi / 2+(\omega / \nu) d) \operatorname{sgn} \omega}+Z^{2}\left(e^{-i \varphi \operatorname{sgn} \omega}-e^{-4(\omega / \nu) a \operatorname{sgn} \omega}\right) \operatorname{sgn} \omega\right] e^{(i p-(\omega / \nu))\left(x-x^{\prime}\right)} \\
& +\left[-\gamma^{-\operatorname{sgn} \omega}\left(1+Z^{2}\right)\left(\gamma^{+} e^{i \varphi / 2+(\omega / \nu) d}-\gamma^{-} e^{-i \varphi / 2-(\omega / \nu) d}\right) e^{(i \varphi / 2-(\omega / \nu) d) \operatorname{sgn} \omega}\right. \\
& \left.\left.+Z^{2}\left(e^{i \varphi \operatorname{sgn} \omega}-e^{-4(\omega / \nu) a \operatorname{sgn} \omega}\right) \operatorname{sgn} \omega\right] e^{-(i p-(\omega / \nu))\left(x-x^{\prime}\right)}\right\}+g\left(x+x^{\prime}\right)
\end{aligned}
$$

where the last term $g\left(x+x^{\prime}\right)$ does not contribute to the current as discussed before. 


\section{JOSEPHSON CURRENT IN A SNINS STRUCTURE}

Josephson current in an SNS structure with barrier (SNINS contact) is found from expression (3),

$$
J=\left.\frac{i e T}{m(2 \pi)^{2}} \sum_{\omega} \int d^{2} k_{\perp}\left(\partial_{x^{\prime}}-\partial_{x}\right) G_{\omega}^{1}\left(x, x^{\prime} ; k_{\perp}\right)\right|_{x^{\prime} \rightarrow x}
$$

When the Green function (27) is substituted into Eq. (28), the Josephson current comes out as follows:

$$
J=\frac{8 e m^{2} T \Delta^{2}}{2 \pi} \sum_{\omega>0} \int_{0}^{v_{F}} v d v \frac{\sin \varphi}{\left(1+Z^{2}\right)\left[(\omega+\Omega)^{2} e^{2(\omega / \nu) d}+(\omega-\Omega)^{2} e^{-2(\omega / \nu) d}\right]+2 \Delta^{2}\left[\cos \varphi+Z^{2} \cosh (4 \omega a / v)\right]}
$$

with $\Omega=\sqrt{\omega^{2}+\Delta^{2}}$ and $Z=V / v$. This expression can be put in a simpler form by using the dimensionless variables, $y$ $=v_{F} / v, \tilde{a}=a / \xi, \widetilde{d}=d / \xi, \tilde{\omega}=\omega / \Delta$, barrier strength $Z_{0}=V / v_{F}\left(\xi=\hbar v_{F} / \Delta\right.$ is the coherence length), as

$$
J=4 \pi J_{0} \frac{T}{\Delta} \sum_{\omega>0} \int_{1}^{\infty} \frac{d y}{y^{3}} \frac{\sin \varphi}{\left(1+Z_{0}^{2} y^{2}\right)\left[(\tilde{\omega}+\widetilde{\Omega})^{2} e^{2 \tilde{\omega} y \tilde{d}}+(\tilde{\omega}-\widetilde{\Omega})^{2} e^{-2 \tilde{\omega} y \tilde{d}}\right]+2\left[\cos \varphi+Z_{0}^{2} y^{2} \cosh (2 \tilde{\omega} y \tilde{a})\right]},
$$

where

$$
\begin{aligned}
& J_{0}=e v_{F} N_{F} \Delta, \\
& N_{F}=p_{F} m / \pi^{2},
\end{aligned}
$$

$N_{F}$ is the density of states at the Fermi level per unit volume.

At $T=0$, the current becomes

$$
J=2 J_{0} \int_{0}^{\infty} d \tilde{\omega} \int_{1}^{\infty} \frac{d y}{y^{3}} \frac{\sin \varphi}{\left(1+Z_{0}^{2} y^{2}\right)\left[(\tilde{\omega}+\widetilde{\Omega})^{2} e^{2 \tilde{\omega} y \tilde{d}}+(\tilde{\omega}-\widetilde{\Omega})^{2} e^{-2 \tilde{\omega} y \tilde{d}}\right]+2\left(\cos \varphi+Z_{0}^{2} y^{2} \cosh (2 \tilde{\omega} y \tilde{a})\right]} .
$$

In the absence of a barrier, Green function $G_{\omega}^{1}\left(x, x^{\prime} ; k_{\perp}\right)$ in Eq. (27) for $-d<x^{\prime}<x<0$ reduces to the following form:

$$
G_{\omega}^{1}\left(x, x^{\prime} ; k_{\perp}\right)=\frac{i}{v} \operatorname{sgn} \omega\left(\frac{e^{(i p-\omega / v)\left|x-x^{\prime}\right|}}{1+\eta(\omega) e^{[i \varphi+2 \omega / \nu d] \operatorname{sgn} \omega}}+\frac{e^{-(i p-\omega / v)\left|x-x^{\prime}\right|}}{1+\eta(\omega) e^{[-i \varphi+2 \omega / \nu d] \operatorname{sgn} \omega}}\right)
$$

where $\eta(\omega)=(\Omega+|\omega|) /(\Omega-|\omega|)$, yielding the Josephson current

$$
J=\frac{8 e m^{2} T}{2 \pi} \int_{0}^{v_{F}} v d v \sum_{\omega>0} \sum_{n=1}^{\infty}(-1)^{n+1}\left(\frac{\sqrt{\omega^{2}+\Delta^{2}}-\omega}{\sqrt{\omega^{2}+\Delta^{2}}+\omega}\right)^{n} e^{-2 \omega n d / v} \sin n \varphi .
$$

$J(\varphi)$ reduces to a saw-tooth function at $T=0$ when all the harmonics contribute,

$$
J=\frac{J_{0}}{6}\left(\frac{\xi}{d}+o\left(\frac{\xi^{2}}{d^{2}}\right)\right) \varphi, \quad-\pi<\varphi<\pi
$$

which is inversely proportional to $d / \xi$ for $d \gg \xi$, and can be written in a more transparent form

$$
J=n e v_{s}
$$

where $v_{s}=(\hbar / 2 m)(\varphi / d)$ is the superfluid velocity and $n$ is the normal electron density.

In the absence of a barrier $\left(Z_{0}=0\right)$, Eqs. (30) and (32) yield the current-phase relationships shown in Fig. 2, where $J_{c 0}$ is the critical current for the parameters $d=10, Z=0$, and $T=0$ [see Eq. (35)],

$$
J_{c 0}=\frac{J_{0} \pi}{60}
$$

The dependence reduces to a saw-tooth function at $T=0$, and converges to a sinusoidal form as the temperature is increased.

At $Z_{0}=0$ and $d \gg \xi_{T}\left(\xi_{T}=\hbar v_{F} / \pi T\right)$ only the lowest harmonic survives, thus the current converges to a sinusoidal form

$$
J=2 e v_{F} N_{F} \frac{v_{F}}{d} \frac{\sqrt{\pi^{2} T^{2}+\Delta^{2}}-\pi T}{\sqrt{\pi^{2} T^{2}+\Delta^{2}}+\pi T} e^{-2 d / \xi_{T}} \sin \varphi
$$

At low temperatures, when $\Delta \gg T$, this expression further simplifies to 


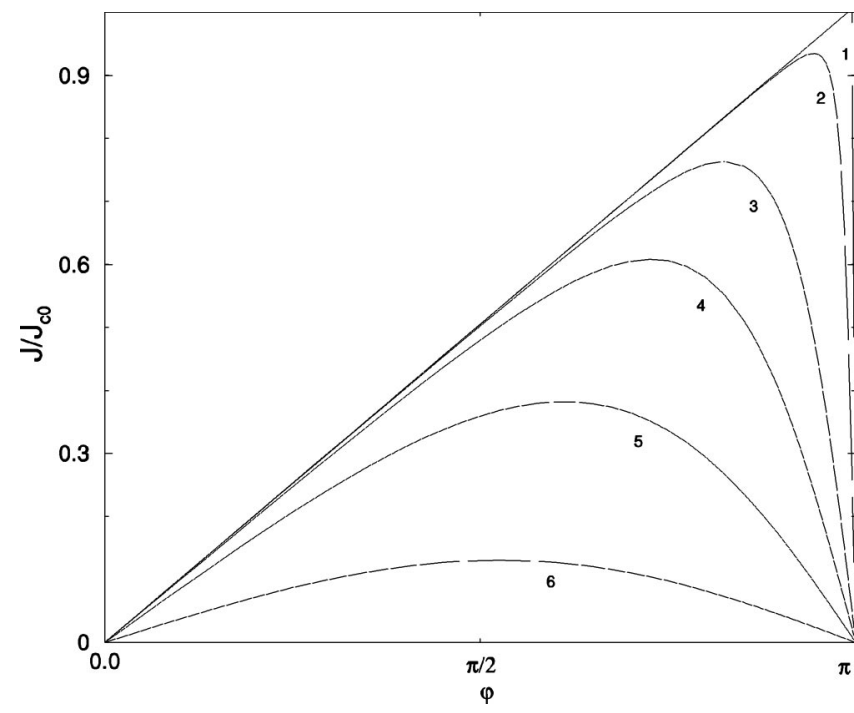

FIG. 2. Current-phase relationship at $d=10 \xi, Z=0$, and various temperatures. (1) $T=0$; (2) $T=0.001 \Delta$; (3) $T=0.005 \Delta$; (4) $T=0.01 \Delta$; (5) $T=0.02 \Delta$; (6) $T=0.04 \Delta$. $J_{c 0}$ is given by Eq. (37).

$$
J=2 e v_{F} N_{F} \frac{v_{F}}{d} e^{-2 d / \xi_{T}} \sin \varphi
$$

We can trace this behavior in Fig. 3; however one should be cautious that Eq. (39) breaks down as $T \rightarrow 0$.

In Fig. 4 we see that the shape of current-phase relationship is quite sensitive to non-Andreev scattering as well as to temperature. Even at $T=0$, the existence of non-Andreev scattering wipes away the higher harmonics, thus resulting in a perfect sinusoidal current-phase relationship at $Z_{0} \gg 1$ [see Eq. (30)],

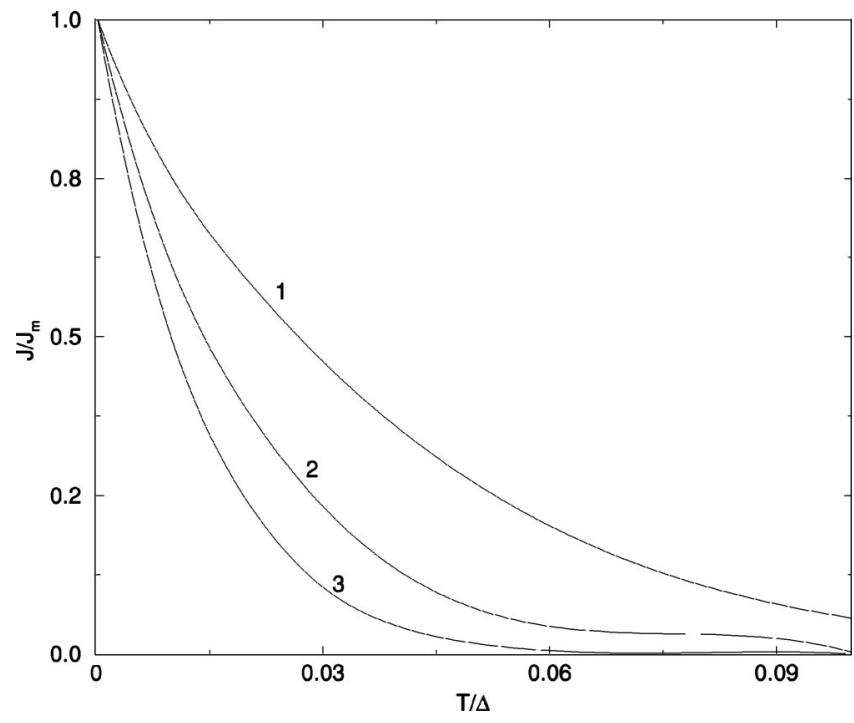

FIG. 3. Temperature dependence of the critical current at $Z_{0}$ $=0, a=0$ ( $a$ is the distance of the barrier from the center of the normal region, see Fig. 1) and various thicknesses of the normal region. (1) $d=5 \xi$; (2) $d=10 \xi$; (3) $d=15 \xi$. $J_{m}$ is the value of the critical current for the corresponding value of $d=\{5,10,15\}$ [see Eq. (35)], with respect to which each of the graphs is normalized.

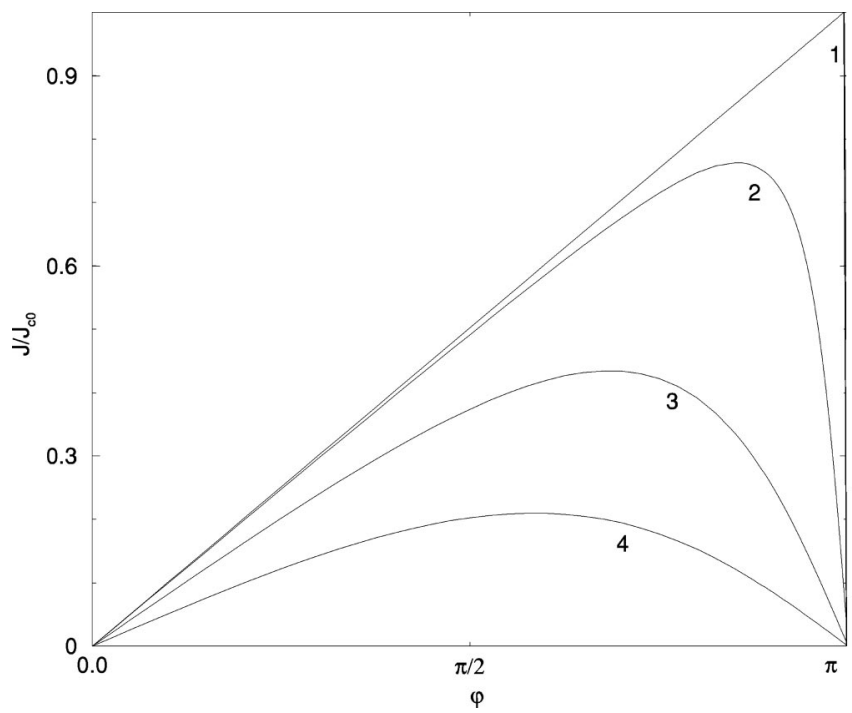

FIG. 4. Current-phase relationship at $T=0, d=10 \xi, a=0$, and various transparencies. (1) $1 / Z_{0}^{2}=\infty$; (2) $1 / Z_{0}^{2}=200$; (3) $1 / Z_{0}^{2}=10$; (4) $1 / Z_{0}^{2}=2 . J_{c 0}$ is given by Eq. (37).

$$
\begin{aligned}
J= & \frac{4 \pi T J_{0}}{\Delta Z_{0}^{2}} \sum_{\omega>0} \int_{1}^{\infty} \frac{d y}{y^{5}} \\
& \times \frac{\sin \varphi}{(\tilde{\omega}+\widetilde{\Omega})^{2} e^{2 \tilde{\omega} y \tilde{d}}+(\tilde{\omega}-\widetilde{\Omega})^{2} e^{-2 \tilde{\omega} y \tilde{d}}+2 \cosh (2 \tilde{\omega} y \tilde{a})},
\end{aligned}
$$

from which we infer that the temperature dependence has the same form at all $Z_{0} \gg 1$. In the tunneling limit $Z_{0} \gg 1$ and at $d \gg \xi_{T}=v_{F} / \pi T$, the current becomes independent of the barrier position,

$$
J=\frac{1}{Z_{0}^{2}} 2 e v_{F} N_{F} \frac{v_{F}}{d} e^{-2 d / \xi_{T}} \sin \varphi,
$$

which differs from that of a pure SNS junction by the transparency factor $1 / Z_{0}^{2}$ [Eq. (39)]. On the other hand, the situation is quite different for small $Z_{0}$ values; the critical current becomes immune to temperature changes as $Z_{0}$ increases, at low temperatures (see Fig. 5). Also in Fig. 6, in the tunneling regime $Z_{0} \gg 1$, the linear dependence of critical current on the barrier transparency is observed.

A crucial observation is the very weak dependence of Josephson current on the position of the barrier, however, the current still has a maximum when the barrier is at the middle of the normal region (Fig. 7), and further the barrier is less sensitive to the position of the barrier at higher temperatures.

When the width of the normal region is taken to be zero, i.e., for $d=0$, the SIS case is recovered. The Josephson current (29) for $d=0$ reduces to a form interpolating between the tunneling regime ${ }^{2}$ and the constriction-type barriers ${ }^{4,12}$

$$
J=\frac{e m^{2} T}{\pi} \sum_{\omega>0} \int_{0}^{v_{F}} v d v \frac{\Delta^{2} \sin \varphi}{\omega^{2}\left(1+Z^{2}\right)+\Delta^{2}\left(\cos ^{2} \varphi / 2+Z^{2}\right)}
$$




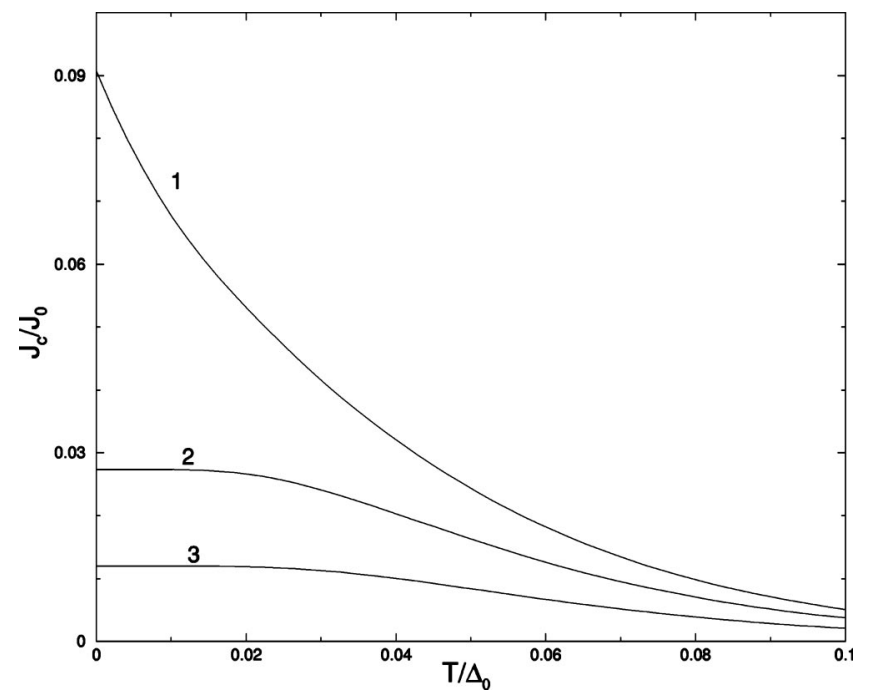

FIG. 5. Temperature dependence of critical current at low temperatures for a normal region of thickness $d=5 \xi$ with a barrier positioned at $a=0$, at various barrier strengths. (1) $Z_{0}=0$; (2) $Z_{0}$ $=0.5$; (3) $Z_{0}=1$.

\section{CONCLUSION}

The Josephson effect in the SNS structure with a $\delta$ barrier in an arbitrary position within the barrier is investigated. The Green functions are calculated by solving the Gorkov equations, while the algebra is greatly simplified by quasiclassical approximations. However, unlike in a pure SNS junction, the whole system cannot be handled in a quasiclassical sense since the Fermi momentum is not conserved throughout the system, due to normal scattering. Andreev scattering is a momentum conserving process, so the quasiclassical approximation works only at the regions in which there is no normal scattering. On the other hand, in a pure SNS junction, electrons and holes with positive and negative momenta do not get mixed up, so the entire system may be treated quasiclassically.

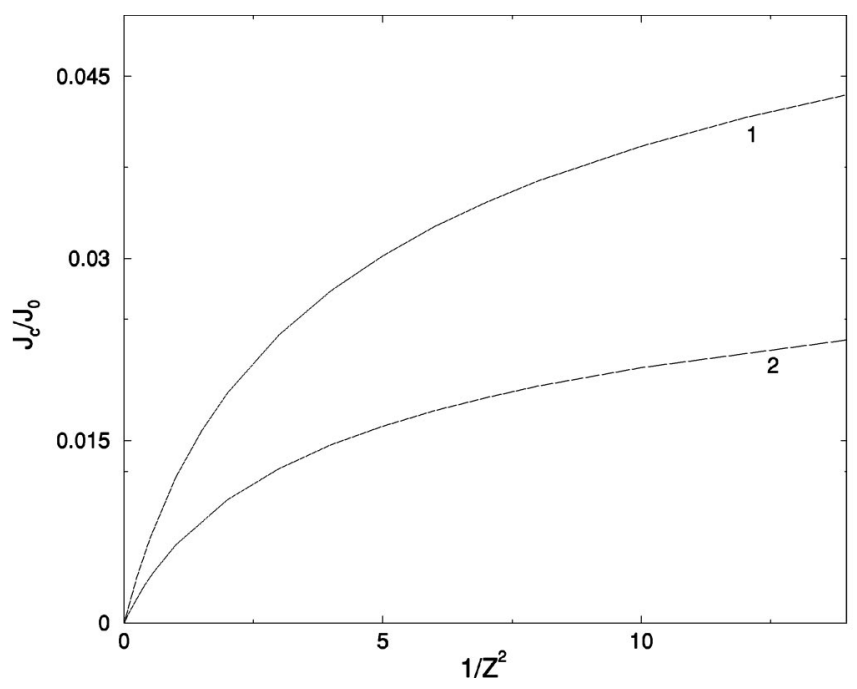

FIG. 6. The dependence of critical current on the barrier transparency, $1 / Z^{2}$, at $T=0$. (1) $d=5 \xi$; (2) $d=10 \xi$. The barrier is taken to be at the middle of the normal region.

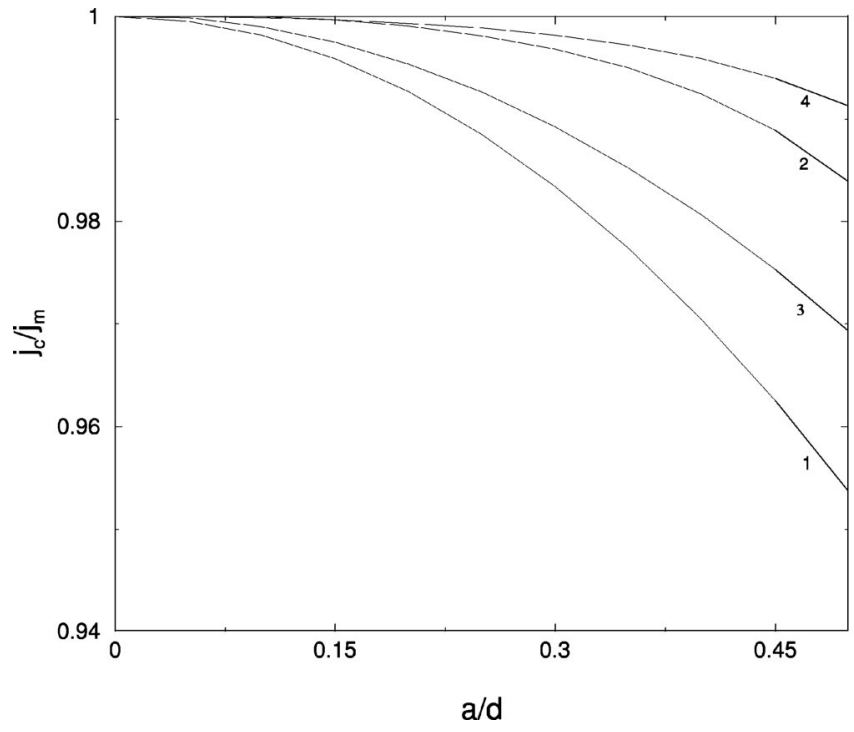

FIG. 7. The dependence of current on the position of the barrier for $d=10 \xi$ and barrier location $a=0$, at various barrier strengths and temperatures. (1) $Z=100, T=0$; (2) $Z=100, T=0.1 \Delta_{0}$; (3) $Z=1, T=0$; (4) $Z=1, T=0.1 \Delta_{0} . J_{m}$ is the critical current at $T$ $=0$ and $a=0$, for the corresponding value of $Z_{0}$, with respect to which each graph is normalized [see Eq. (32)].

At zero temperature, in a pure SNS structure when $d \gg \xi$ the current is proportional to the gradient of the phase across the normal region, i.e., $\varphi / d$ [Eq. (34)], which is proportional to the superfluid velocity. In fact an effective gap of order of $\hbar v_{F} / d$ develops which sets new length and energy scales [see Eqs. (35) and (39)]. In this case the Cooper pairs are transported via multiple Andreev reflections, as a result of which all the reflective harmonics contribute [Eq. (34)], resulting in a saw-tooth phase dependence of the current. However, the introduction of normal scattering simulates a tunneling junction behavior, and this time the prominent mechanism of supercurrent becomes that of the tunneling of Cooper pairs, hence the phase dependence of the current becomes sinusoidal, even at $T=0$. At $T=0$ case, the Cooper pair amplitude in the normal region in an SN proximity junction decays on a power law on thickness of normal region, $1 / d$, while at $d \gg \xi_{T}=v_{F} / T$ and $T \ll T_{c}$ the amplitude decays exponentially as $\exp \left(-d / \xi_{T}\right),{ }^{21}$ which point out to the fact that the critical current in the SNS structure is proportional to the Cooper pair amplitude. In a real junction, superconductive and the normal regions are those of different metals hence inevitably there is normal scattering at the SN interface, and further there may be a normal scattering due to impurities and imperfections in the bulk of $N$ electrode as well as on the SN interfaces. However, a general behavior may be expected when the barrier is strong, $Z_{0} \gg 1$, where $Z_{0}=V / v_{F}$ is the effective strength of the barrier. In this case, the current acquires sinusoidal phase dependence since tunneling becomes the dominant transport mechanism. However when the barriers are weak, the existence of multiple barriers may lead to complex behaviors. Further the position of the barrier does not lead to significant change in the behavior of 
the junction (see Fig. 7), i.e., the conclusive effect of the barrier arises from its strength.

The potential barrier inserted in the normal metal makes the non-Andreev scattering of quasiparticles in the SNS structure possible, and represents a model for more general situations when such scattering becomes effective, e.g., dissimilarity between $S$ and $N$ electrodes due to the difference in their densities of normal states, the values of chemical potential, etc., which are unavoidable in real superconductive Josephson structures. In our model, these factors are incorporated through the additional parameters of the contact, $Z_{0}$, which was first introduced by Blonder et al. in their theory of diffusive SNS junctions. ${ }^{22}$

In our formulation, the usual SNS, SIS and SCS cases, those of the normal-metal barrier, the tunneling barrier, and the constriction-type barrier are recovered in the corresponding limiting cases.
${ }^{1}$ B.D. Josephson, Phys. Lett. 1, 251 (1962); Adv. Phys. 14, 419 (1965)

${ }^{2}$ V. Ambegaokar and A. Baratoff, Phys. Rev. Lett. 10, 486 (1963).

${ }^{3}$ I.O. Kulik and I.K. Yanson, The Josephson Effect in Superconductive Tunneling Structures (Izdatel'stvo Nauka, Moscow, 1970) (Israel Program for Scientific Translations, Jerusalem, 1972).

${ }^{4}$ I.O. Kulik and A.N. Omelyanchouk, Pis'ma Zh. Eksp. Teor. Fiz. 21, 216 (1975) [JETP Lett. 21, 96 (1975)]; Fiz. Nizk. Temp. 3, 945 (1977) [Sov. J. Low Temp. Phys. 3, 459 (1977)]; Fiz. Nizk. Temp. 4, 296 (1978) [Sov. J. Low Temp. Phys. 4, 142 (1978)].

${ }^{5}$ A.F. Andreev, Zh. Eksp. Teor. Fiz. 49, 655 (1965) [Sov. Phys. JETP 22, 455 (1966)].

${ }^{6}$ I.O. Kulik, Zh. Eksp. Teor. Fiz. 57, 1745 (1969) [Sov. Phys. JETP 30, 944 (1970)].

${ }^{7}$ I.O. Kulik, Dr. Sci. Thesis (in Russian), B. Verkin Inst. Low Temp. Phys. and Engng., Kharkov, 1972; I. O. Kulik (unpublished). In these references, a numeric error in Ref. 6, first noticed by Ishii (Ref. 8), was corrected. The papers stress on the fact that the Andreev quantization and the Josephson effect represent, in fact, a same physical phenomenon, i.e., the quantization cannot exist when the phase coherence is broken (e.g., by fluctuation) and, accordingly, there is no Josephson supercurrent when quasiparticles are not quantized.

${ }^{8}$ C. Ishii, Prog. Theor. Phys. 44, 1525 (1970); 47, 1464 (1972).

${ }^{9}$ J. Bardeen and J.L. Johnson, Phys. Rev. B 5, 72 (1972).
${ }^{10}$ A.V. Svidzinsky, T.N. Antsygina, and E.N. Bratus, Zh. Eksp. Teor. Fiz. 61, 1612 (1971) [Sov. J. Low Temp. Phys. 10, 131 (1973)].

${ }^{11}$ A. Furusaki, H. Takayanagi, and M. Tsukada, Phys. Rev. B 45, 10563 (1992); Solid State Commun. 78, 299 (1991).

${ }^{12}$ A. Furusaki, in Physics and Applications of Mesoscopic Josephson Junctions, edited by H. Ohta and C. Ishii (The Physical Society of Japan, Tokyo, 1999), p. 79.

${ }^{13} \mathrm{H}$. Takayanagi, in Physics and Applications of Mesoscopic Josephson Junctions (Ref. 12), p. 233.

${ }^{14}$ W.L. McMillan, Phys. Rev. 175, 559 (1968).

${ }^{15}$ P.G. de Gennes, Superconductivity of Metals and Alloys (Benjamin, New York, 1966).

${ }^{16}$ G. Eilenberger, Z. Phys. 214, 195 (1968).

${ }^{17}$ A.V. Zaitsev, Zh. Eksp. Teor. Fiz. 86, 1742 (1984) [Sov. Phys. JETP 59, 1015 (1984)].

${ }^{18}$ M.Y. Kuprianov and V.F. Lukichev, Zh. Eksp. Teor. Fiz. 94, 139 (1988) [Sov. Phys. JETP 67, 1163 (1988)].

${ }^{19}$ A.A. Golubov, F.K. Wilhelm, and A.D. Zaikin, Phys. Rev. B 55, 1123 (1997).

${ }^{20}$ A.A. Abrikosov, L.P. Gorkov, and I.E. Dzyaloshinski, Methods of Quantum Field Theory in Statistical Physics (Dover New York, 1975).

${ }^{21}$ P.G. de Gennes and G. Deutscher, in Superconductivity, edited by R. D. Parks (Marcel Dekker, 1969).

${ }^{22}$ G.E. Blonder, M. Tinkham, and T.M. Klapwijk, Phys. Rev. B 25, 4515 (1982). 